\title{
Beiträge zur Kenntnis der Flora von Hokkaido.
}

\author{
Von \\ H. Takeda. \\ (Fortsetzung. $)^{1)}$
}

61. Matricaria discoidea DC. Prodr. VI, p. 50.-Ledeb. Fl. Ross. II, p. 544.-Regel et Til. Fl. Ajan. p. 102.-Miyabe, Fl. Kuril. p. 242.

Hab. Yezo: Secus vias oppidi Nemuro, abundans. (H. TAKEDA!) VIII. 1909).

Die Pflanze ist in den Kurilen sowie auf Sachalin bekannt, wo wie auf Yezo eingebürgert ist. Auf Sachalin müssen die Russen die Pflanze zum $Z$ wecke der Medizin kultiviert haben.

62. Fragaria neglecta LindeM. in Bull. Soc. Imp. Nat. Mosc. XXXVII, 2 (1865), p. 220.-Freyn, in Oesterr. Bot. Zeitschr. 1895, p. 312.

Fragaria collina Auctor plur. (non EHRH.) sec Lindem.

\Calyce fructus adpresso, pubescentia petiolorum patentissimo, pedunculorum pacente (marcescentium interdum adpresso); foliis supra pubescentibus, subtus sericeis ; sporophoro depresso, nutante, obtuso maturo adhærente. Differt a $F r$. vesca et ab elatiore calyce fructus adpresso non reflexo, a $F r$. collina pubescentia pedunculorum patente nec adpressa《 (Lindem. 1. c.). Staminibus capitulo carpellorum longioribus ( $3 \frac{1}{2} \mathrm{~mm}$. long.).

Nom. Japon. Yezono-Kusaichigo (nom. Ainu: Frepp).

Hab. Yezo : Circa oppid. Kushiro, in sylvis (U. Faurie! 24. VI. 1890, n, 5310); in pratis Haruturn prope Kushiro (H. TAKeda! VII. 1909); Tamoshiri, prope oppid. Nemuro (H. TAKEDA! VII. 1909 nec non in pratis collibusque regionis orientalis frequentissima.

1) Vgl. Tôkyô Bot. Mag. XXIV, S. 7, S. 131, S. 1ố6, S. 17ó, S. 243. S. 253. 
Kurile: in porto Shakotan, ins Shikotan (M. Aizawa! 13. VI. 1900 ; H. TAKEDA! 16. VII. 1909); fere per totam ejusd. insul. distributa est.

63. Fragaria vesca Linn. Sp. P1. p. 494, parte.-KocH, Syn. F1. Germ. Helv. ed. 2, p. 234.-Sḱr. in DC. Prodr. II, p. 569.Aschers. u. Graebn. Syn. Mitteleurop. F1. VI, 1, p. 649.

Fragaria elatior Auct. plur. japon. non EHRH.

Icon. Hayne, Arzn. Gew. IV, tab. 26.--Irnuma, Sômoku Dzusetsu.

Pubescentia pedicellorum sub anthesi adpressa, tum post anthesin patentia; calyce reflexo; staminibus capitulum carpellorum non superantibus; acheniis numerosioribus a specie præcedenti dignoscitur.

Nom. Japon Shirobanano-Hebiichigo.

Hab. Yezo: in pratis herbeosis Maruyama, prope Satporo (H. TAKEDA! 20. VI. 1907).

Daß diese Erdbeereart auf Yezo einheimisch ist, ist eher fraglich. Die Pflanze kommt in der Waldregion Mittelnippons häufig vor. Aesserst selten habe ich die Monstrosität pinnata ${ }^{1)}$ gesehen.

64. Trientalis europæa Linn. $\beta$ arctica LedeB. Fl. Ross. III, p. 25.-Herd. Pl. Radd. IV, 1, p. 132.-Miyabe, F1. Kuril. p. 251. -Hayata, in Tokyo Bot. Mag. XVII. p. 34.

Trientalis arctica Fisch ex Hook. F1. Bor.-Am. II, p. 121.DC. Prodr. VIII, p. 59.-PIPER, Fl. Washnigt. p. 447.

Trientalis europæa Cnam. et Schlechtol. in Linnæa, I, p. 224.-Hook, et Arn. in Belchey's Voy. p. 116, excl. Syn. Engl. Bot.-Bongard, Véget. ins: Sitcha, p. 160.

Alsinanthemos caulibus aleimo foliosis, foliis alternis. GMEI.IN, Fl. Sibir. IV, p. 116, n. 86.

Nom. Japon. Ko-Tsumatori-sô.

Hab. Yezo: Otsu, prov. Tokapchi (S. TANouchi ; 14. VI. 1881) ; Tumoshiri, prope oppid. Nemuro (M. Nakamura! 26 VI. 1885; H. TAKEDA！5. VII. 1909).

1) Fragaria vesca LINN monstr. pinnata mihi f liis radicalibus nonnulis bijugopinnatis (Hab. Nikko, BISSET, n. 1054, hb. Kew.). 
Kurile: Shakotan, ins. Shikotan (T. KaWAKAMI! 1. VIII. 1898 ; H. TAKEDA ! 16. VII. 1909).

65. Anaphalis yedoensis Maxim. in Mél. Biol. XI, p. 235. Gnaphalium yedoense Franch. et SAv. Enum. Pl. Japon. II, p. 464 .

Nom. Japon. Kawara-Hôko.

Hab. Yezo : circa Satporo (U. FaURIe! V. 1885, n. 119, 4. IX. 1886, n. 1305 ; H. TAKEDA! 26. IX, 1908).

66. Scutellaria scordiifolia Fischer.

Forma puberula Komar. Fl. Mansh. III, p. 344.

Scutellaria galericulata s. pubescens Reger, Tentam. Fl. Ussuro n. 388.

Nom. Japon. Yezo-Namiki-sô.

Hab. Yezo: Haruturu, prope oppid. Kushiro (H. TAKeda! 18. VIII. 1909); in herbosis Okchishi. prov. Nemuro (H. TAKEdA ! 10. VIII. 1909).

Kurile: Anama, ins. Shikotan (M. Arai! 19. VIII. 1909).

Forma pubescens Komar. 1. c.

Scutellaria galericulata $\beta$. pubescens REGEL, 1. c.

Scutellaria scordiifolia var: pubescens MiQ. Prolus. Fl. Japon. p. 42 .

Scutellaria scordiifolia var. hirta Fr. SchM. Reis. Amurl. Sachalin, p. 165.

Scutellaria galericulata forma hirta KoInzumi, Pl. Sachalin. NAKahara. p. 104.

Nom. Japon. Namiki-sô.

Hab. Yezo: circa Kushiro (M. Uyeda!)

Kurile: Anama, ins. Shikotan (M. AraI! VIII. 1909).

Sachalin: loco non indicato (FR. Schmidt!)

67. Conioselinum kamtschaticum Rupr. Rev. Umbell. Kamtschatsk. in Beitr. zum Pflanzenk. russ. Reichs. XI, p. 22. -Fr. Schm. Reis. Amurl. Sachalin, p. 135-KoIdzumi, Pl. Sachal. Nakahara. p. 97.

Seseli Libanotis var. sibirica Mryabe, in Batch. and MryaBE, Ainu Econom. Pl. in Transact. Asiat. Soc. XXI, p. 205, n. 15 , nec DC. 
Nom. Japon. Karafuto-Ninjin, Iwa-Ninjin, (nom. Ainu. Upeu).

Hab. ad rupes littoris maritimi frequens. Yezo : circa Kushiro (M. Uyeda !); Nemuro (H. Takeda !).

Kurile: in insulis Shikotan (!); Kunnashiri (!); Eturup (!); Urup (!); Shashikotan (!).

Die Wurzel der Pflanze ist von Ainu-Bewohner als Medizin hochgeschätzt.

68. Fritillaria camschatcensis Ker-GawL. in. Bot. Mag. sub. tab. 1216.-Kunth, Enum P1. IV. p. 25-REgel, Gartenfl. sub. tab. 173.-Mig. Prolus. F1. Japon. p. 322.-Franch. et Sav. Enum. Pl. Japon. II, p. 62.-Wright, in Journ. Linn. Soc. XXXVI, p. 136.-Matsum. Index Pl. Japon. II, 1, p. 196.

Lilium camschatcense Linn. Sp. Pl. p. 303 ; Cod. n. 2392.Willd. Sp. P1. II, p. 89.

Fritillaria kamtschatcensis Fisch. ex Hook. Fl. Bor.-Amer. II, p. 181, tab. 139.-BAKER, in Journ. Linn. Soc. XIV, p. 258. -Fr. Schm. Reis. Amurl. Sachal. p. 186-Miyabe, Fl Kuril. p. 265.

Nom. Japon. Kuro-Yuri.

Hab. Yezo : circa Satporo (H. Takeda! VI. 1906 ; VI. 1907 ; V. 1908); Chitose, prov. Iburi (K. Miyabe! 10. VI 1884) ; Perufune, prov. Tokapchi (K. Mryabe! 24. VI. 1884); in tractu Akan, prov. Kushiro (S. ITô! VI. 1895); Oraporo, prov. Hidaka (Togano! 26. V. 1895); Omsari, prov. Kitami (Togano ; VI. 1896).

Kurile: Shakotan. ins. Shikotan (T. Ishikawa! 23. VI. 1894); Zembekotan, prope pagum Tomari, ins. Kunnashiri (H, TANaka! anno 1893); Onnepet, ins. Eturup (K. Jim Bô! 9. VI. 1891); Rupet, ejusd. ins. (Sh. Yokoyama! 24. VI. 1893); inter Tôro et Porosu, ejusd. ins. (Sh. Yokoyama! VII. 1893); Porosu ('T. Kawakami! 7. VIII. 1897); Naipo, ejusd. ins. (M. AIzawa! 15. VI. 1900); Shibeturo, ejusd. ins. (K. Jim Bô! 13. VI. 1891); Tukotan, ins. Urup (K. Uchida! anno 1891; T. Ishikawa! 23. VII. 1894); Suisanwan, ejusd ins. (K. Uchida! 5. VI. (1891); ad lacu Tukotan et secus fl. Tukotan (K. Jimbô! VI. VI. 1891); in porto Broton, ins. Shimushir (Kr DAMA! VI. 1893); in insula Rashawa (Kопммa! VII. 1893).

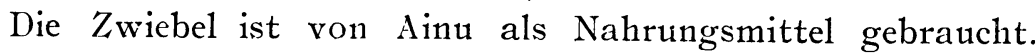
Die Pflanze kommt auch auf Hochgebirgen Nippons nicht selten 
vor, wo sie in der Regel nicht üppig ist und nur eine selten zwei Blïten trägt.

69. Tofieldia Okuboi Makino, in Tôkyô Bot. Mag. XII (1898), p. 42.

Nom. Jap. Hime-Iwa-shôbu.

Hab. Kurile: in monte Atoiya, insulæ Eturup (T. Kawakami 11. VIII. 1898).

Neu für die Flora von Hokkaidô. Obgleich kommt die Pflanze in Hokkaidô sehr selten vor, wird sie auf hohen Gebirgen Nippons manchmal angetroffen.

70. Tofieldia nutans Willd. ex Schultes Syst. VII, p. 1573 in obs.-Matsum. Index Pl. Japon. II, p. 215.

Nom. Japon Chishima-Zekishò.

Hab. Yezo : ad fl. Nukapira, tract. Sarn. pror. Hidaka. (СH. YeNDô! 27. IX. 1895); secus Yaramap, tribut. Perupnei, prov. Tokapchi (K. Jimвô! 1891); in apice montr Rishiri (W. Hirose ! 3. VIII. 1896); in monte Tokapchi ( $\mathrm{Sh}$. Suganuma! 16. VII. 1890).

Kurile: ad rupes Anama, ins. Shikotan (H. TAKEDA! 24. VII. 1909); in rupibus ad ostium fl. Profect, ejnsd. ins. (H. TAKEDA! 23. VII. 1909); in summitate montis s. m. 1000 ped. in porto Moikeshi, ins Eturup (T. Ishikawa! 18. VII. 1890); insula Sakkojiwa. Eturup. ('T. Kawakami! 27. VIII. 1898).

71. Trillium Tschonoskii Maxim. in Mél. Biol. XI, p. 863. -Yatabe, in Tôkyô Bot. Mag. VII. (1893), p. 175, tab. 7.

Nom. Japon. Shirobana-no-Enreisô.

Hab. Yezo: ad decurs. superior. fl. Hacham, prope Satporo (H. TAKEDA! 13. V. 1909); in Sylvis Moiwa, prope Satporo (H. TAKEDA! 16. V. 1909 ; 23. V· 1907).

72. Trillium kamtschaticum PaLL. ex Pursh, Fl. Amer. Sept. I, p. 246, sub T. obovato.-Miyabe, Fl. Kuril. p. 265.Matsum. Index Pl. Japon. II, 1, p. 217.

Trillium obovatum Auct. plur. exel. pl. amer.

Nom. Japon. Obana-no-Enrei-sô.

Hab. Yezo: circa Satporo locis humidis (M. Oguma! 1905; (H. 
TAKeda 1907, 1908, 1909); Tôbets, prov. Oshima (K. Miyabe et Y. Tokubechi! 13. VII. 1890); Poromoi, prov. Ishikari (E. Odagiri ! 21. VI. 1893); in pago Notto, prov. Shiribeshi (N. IsHikawa! 1. VII. 1897); secus riverlos. in pago Shiripet, prov. Shiribeshi (N. IsHIKawa! 14. V. 1897); Priroro, prov. Tokapchi (K. Mryabe! 23. VI. 1884); Oraporo, prov. Hidaka (HorI! VI, 1896); in tractu Meakan, prov. Kushiro (S. Itô! V. 1893); Tumoshiri, prov. Nemuro (M. Nakamura! 26. V. 1885); in monte Rishiri, ins. Rishiri (W. Hirose ! 20. VIII. 1896); in pago Shikin, prov. Iburi E. OdAGIRI! V. 1894).

73. Veratrum nigrum LiNN var. japonicum BAKER, in Journ. Linn. Soc. XVII, p. 472.-MATsum. Index Pl. Japon. II, 1. p. 218.

Differt a V. Maackii Regel foliis oblongis nec auguste lanceolatis, paniculis densissimis nec sparsis, pedicellis floribus aequantibus nec subtriplo superantibus nec gracilibus, rachi lanato-pubescenti.

Nom. Jaron O-Shuro-sô.

Hab. Yezo : Hakodate (C. WiLford! 1859, n. 1004; AlbReCht ! 1861).

Die Pflanze kommt auch in Nordnippon vor.

74. Veratrum stamineum Maxim. in Bull. Soc. Imp. Sc. St. Petersb. XV, p. 230.-Franch. et Sav. Enum. P1. Japon. II, p. 90.-BAKER, in Journ. Linn. Soc. XVII, p. 471.

Nom. Japon Ko-Baikei-sô.

Hab. Yezo: in Turfosis inundatis Poromoi, prov. Ishikari (H. TAKEDA! 27. VI. 1906).

75. Dryopteris fragrans SchotT, Gen. Fil. ad tab. 9.Underwood, Our Nat. Ferns, ed. 6, p. 112.-C. Christ. Index Fil. p. 266.

Polypodium fragrans Linn. Sp. Pl. p. 1089.

Aspidium fragrans Sw. Syn. Fil. p. 51.-Willd. Sp. P1. V, p. 253.-Milde, Fil. Europ. At1. p. 117.-Miyabe, Fl. Kuril. p. 274.-Christ, Farnkr. d. Erde, p. 260.

Nephrodium fragrans RICHARD, in Frank1. Narr. Journ. p. 754.-Hoor. et Bar. Syn. Fil. p. 275.-Diels, in Engl. u. Pr. Pflanzenfam. I, 4. p. 173.-Matsum. Index Pl. Japon. I, p. 319. 
Lastrea fragrans PresL, Tentam. Pteridogr. p. 76.

Polystichum fragrans Leder. Fl. Ross. IV, p. 514.

Nom. Japon. Nioi-Shida.

Hab. Yezo : in rupileus Otaru (K. Mryabe! VI. 1886); Osatsube, prov. Oshima (K. Mivabe! 24. VIII. 1896); Mororan (K. Miyabe et Y. Tokubuchi! 27. IV. 1894); in sylvis Moiwa (Y. Tokubuchi! 20. V. 1890 ; H. TAKEDA! 1907, 1908); Abashiri, prov. Kitami (K. MryABE! 16. VI. 1884).

Kurile: Shakotan, ins. Shikotan (K. Miyabe! 28. VII. 1884).

Sachalin : oliis locis (T. Mryake!).

Dieser Farn ist neulich in Zentralnippon $\mathfrak{u}$. zwar auf Yatsugatake aufgefunden worden.

76. Jryopteris Miqueliana C. Christ. Index Fil. p. 278. Aspidium Miquelianum Maxim. apud Franch. et Sav. Enum. Pl. Japon, II, pp. 240, 634.-Matsum. Index P1. Japon. I, p. 288.

Nom. Japon. Narai-Shida.

Hab. Yezo: Makomanai, prope Satporo (K. Mryabe! 20. IX. 1883); Sarurn et Saruru-sando (Y. Tokubuchi! 14 VIII. 1892); Kamuikotan (K. Mryabe! 12. VIII. 1891); in insula parva Okushiri (K. Miyabe et Y. Tokubuchi! 28. VII. 1890); Yuparo, prov. Ishikari (Y. ToKubuchi! 91. VIII. 1893); Garngawa, prope Satporo (K. Miyabe! 9. IX. 1891)

77. Dryopteris Sabæi C. Christ. Index Frl. p. 290.

Aspidium Sabæi Franch. et Sav. Enum. Pl. Japon. II, pp. 259, 632 .

Nephrodium filix-mas var. Sabæi CHrist, in Bull. Boiss. 1899, p. 822.-Matsum. Ind. Pl. Japon I, pp. 319, 384.

Nom. Japon. Miyama-Itachi-shida.

Hab. Yezo : in insula parva Okushiri (K. Mryabe et Y. TokuBUChI! 28. VII. 1892); Ichinowatari, prov. Oshima (K. Mryabe et Y. Tokubcchi! 16. VI. 1890); Esashi, prov. Oshima (Y. Tokubuchi! 8. VIII. 1888).

78. Camptosorus sibiricus Rupr. Distr. Crypt. vasc. Ross. in Beiträge zur Pflanzenk. Russ. Reichs, III, p. 45.-C. CHrist. Index Fil. p. 166. 
Scolopendrium sibiricum Hook. Second Cent. Ferns sub, tab. 35.-Hook. et BaKer, Syn. Fil. p. 248-Matsum. Index Pl. Japon. I, p. 347 .

Antigramma sibirica J. Sm. Hist. Fil. p. 331.

Phyllitis sibirica O. Kтze. Rev. Gen. P1. II, p. 818.

Nom. Japon. Kumonosu-Shida.

Hab. Yezo: Kamuikotan (!); in rupibus calcareis Tosshuyama, prope oppid, Asahikawa (T. MiYake!).

79. Plagiogyria Matsumureana Makino, in Tôkyô Bot. Mag. VIII, (1894), p. 333.-Matsum. Index Pl. Japon. I, p. 332.-C. Christ. Index Fil. p. 496.

Lomaria Matsumureana Makino, 1.c. p. 90 (pars japon.).

Plagiogyria Fauriei Matsum. 1. c. p. 332.-C. Christ. 1.c. p. 495.

Lomatia Fauriei C.hrist, in Bull. Boiss, IV (1896), p. 666.

Blechnum Fauriei Tokubuchi, in Tôkyô Bot. Mag. XIX (1905), p. 231.

Nom. Japon. Yama-Sotetsu.

Hab. Yezo: Satporo (K. Miyabe! VII. 1879); in sylvaticis umbrosis montis Teine, prope Satporo (Y. Toкuвuch ! 9. X. 1894); in montibus Chiriochi, prov. Oshima (K. Mirabe et Y. Tokubuchi! 14. VII. 1890); in insula parva Okushiri, prov. Shiribeshi (K. Mrvabe et Y. Tokubuchi ! 28. VII. 1890.

Dieser Farn wird im Waldgebiete der Gebirgsgegenden Mittelund Nordnippons sehr häufig angetroffen. 